\title{
CARACTERIZACIÓN DE RESIDUOS SÓLIDOS Y DISEÑO DE UN RELLENO SANITARIO MANUAL PARA EL DISTRITO LA YARADA LOS PALOS
}

\author{
CHARACTERIZATION OF SOLID WASTE AND DESIGN OF A MANUAL \\ LANDFILL FOR THE DISTRICT LA YARADA LOS PALOS
}

Richard Sabino Lazo Ramos ${ }^{1}$ Dina Marlene Cotrado Flores ${ }^{2}$ Diego Abel Sequeiros Abarca ${ }^{3}$ Virginia Apaza Pilco ${ }^{4}$ Alejandro Jena Pier Mamani Vargas ${ }^{5}$ Ray Mamani Juli ${ }^{6}$

Información del artículo: Recibido: 08/10/2020 Aceptado: 15/12/2020

${ }^{1}$ Docente escuela de Ingeniería Ambiental, Universidad Privada de Tacna, Perú ${ }^{2}$ Docente escuela de Ingeniería Civil, Universidad Privada de Tacna, Perú ${ }^{3,4,5}$ Escuela de Ingeniería Ambiental, Universidad Privada de Tacna, Perú

${ }^{6}$ Escuela de Ingeniería de civil, Universidad Privada de Tacna, Perú E-mail; ${ }^{1}$ ozalsomar@gmail.com, ${ }^{2}$ dinacotrado@hotmail.com, ${ }^{3} \mathrm{dsa} 30 @$ hotmail.com, 4virmi_21@hotmail.com, 5alejandro.jeanpiermv@gmail.com, 6jhossimar_mamani_juli@hotmail.com 


\section{Resumen}

El estudio de caracterización de los residuos sólidos se realizó en el distrito La Yarada -Los Palos, Provincia de Tacna, con la finalidad de analizar los residuos domiciliarios y no domiciliarios de las viviendas, mercados, centros educativos, tiendas comerciales, ferreterías y comisarias. Se empleó la metodología la descrita por el Ministerio del Ambiente (2018) para determinar la cantidad y características de los residuos sólidos mediante muestreos aleatorios. La generación per cápita de residuos sólidos domiciliarios fue de $0,470 \mathrm{~kg} / \mathrm{hab}$-día, en la composición física se encontró en mayor cantidad, la materia orgánica con $56,77 \%$, la densidad promedio sin compactar fue de $224,682 \mathrm{~kg} / \mathrm{m}^{3}$ y el compactado $305,664 \mathrm{~kg} / \mathrm{m}^{3}$. En cuanto a los residuos no domiciliarios se determinó que las instituciones educativas generan más residuos sólidos con $38,47 \%$, seguido de tiendas de abarrotes con $28,53 \%$, tiendas de fruta con $8,60 \%$, restaurantes $7,21 \%$ y plazas con 4,10 \%. Con la información obtenida se diseñó el relleno sanitario manual aplicando la Guía de diseño, construcción, operación, mantenimiento y cierre de relleno sanitario manual del Ministerio del Ambiente que consta de nueve zanjas de 2 659,2 m3 de residuos sólidos como volumen útil de diseño con una proyección de 10 años para los $24552,7 \mathrm{~m} 3$. Con celdas de 2,4 m de altura, largo superior (Is) de $60 \mathrm{~m}$ ancho superior (as) $30 \mathrm{~m}$ área superior $825 \mathrm{~m} 2$ talud de trinchera (H) 1, talud de trinchera (V) 1, largo inferior (li) 55,2 m, ancho inferior (ai) 25,2 m y área inferior (Ai) 1391,04 m2.

Palabras claves: Caracterización; diseño relleno sanitario manual; residuos sólidos.

\section{Abstract}

The study of the characterization of solid waste was carried out in the district La Yarada -Los Palos, Tacna Province, in order to analyze the home and non-domicile waste of homes, markets, schools, shops, hardware stores and curators. The methodology described by the Ministry of the Environment (2018) was used to determine the quantity and characteristics of solid waste by random sampling. The per capita generation of household solid waste was $0.470 \mathrm{~kg} / \mathrm{hab}$-day, in the physical composition, organic matter was found in greater quantity was $56.77 \%$, the average uncompacted density was 224,682 $\mathrm{kg} / \mathrm{m} 3$ and the compacted $305,664 \mathrm{~kg} / \mathrm{m} 3$. In terms of non-home waste, it was determined that educational institutions generate more solid waste at $38.47 \%$, followed by grocery stores with $28.53 \%$, fruit shops with $8.60 \%$, restaurants $7.21 \%$ and places with $4.10 \%$. With the information obtained, the manual landfill was designed by applying the Ministry of the Environment's Manual Sanitary Design, Construction, Operation, Maintenance and Closure Guide consisting of nine 2,659.2 $\mathrm{m} 3$ ditches of solid waste as a useful design volume with a 10-year projection for the $24552.7 \mathrm{~m} 3$. With cells $2.4 \mathrm{~m}$ high, upper length (Is) $60 \mathrm{~m}$ upper width (as) $30 \mathrm{~m}$ upper area $825 \mathrm{~m} 2$ trench slope (H) 1, trench slope (V) 1, lower length (li) $55.2 \mathrm{~m}$, lower width (ai) $25.2 \mathrm{~m}$ and lower area (Ai) $1391.04 \mathrm{~m} 2$.

Keywords: Characterization; manual landfill design; solid waste. 


\section{Introducción}

A nivel mundial, especialmente en las grandes ciudades de los países de América Latina y el Caribe, el manejo de los residuos sólidos ha representado un problema debido, entre otras cosas, a los altos volúmenes de residuos sólidos generados por los ciudadano (Sáez y Urdaneta, 2014), por ello con la finalidad de cuantificar y conocer las propiedades fisicoquímicas y biológicas de los residuos, la producción per cápita para la toma de decisiones sobre la mejor forma para el aprovechamiento o tratamiento final se han realizado diversos estudios (puerta 2014; Rendón, 2012; Ruiz, 2012), en esa dirección el distrito de la Yarada Los Palos, no es ajena a la problemática, por lo que el estudio de caracterización de residuos sólidos, es el empleo de procedimientos y fórmulas para obtener información de las características cuantitativas de los residuos sólidos del el distrito de La Yarada Los Palos que no posee un adecuado sistema de gestión de residuos sólidos y por tanto un lugar donde disponerlo, ya que su mala disposición en lugares inadecuados puede provocar una serie de enfermedades gastrointestinales, a la piel y respiratorias. Por lo tanto es necesario la caracterización de estos residuos en instituciones restaurantes, mercados, comercios. La información generada puede ayudar a tomar decisiones que conlleven a la mejora de la gestión y manejo por parte de la Municipalidad como del almacenamiento público, recolección y transporte, y además a partir de los resultados del estudio se podrá diseñar un relleno sanitario manual en lugar asignado por el municipio en donde se realizará la disposición final de los residuos. Por otro lado también es necesario implantar acciones de reciclaje para residuos inorgánicos y orgánicos reaprovechables.

\section{Objetivo}

El estudio tiene la finalidad de contribuir con el aporte de información sobre las características de los residuos sólidos para mejorar la prestación de servicios de limpieza pública con enfoque de eco eficiencia.

\section{Metodología}

\section{Determinación de la generación de residuos sólidos domiciliarios}

Según el Instituto Nacional de Estadística e Informática (INEI) para el año 2016 las viviendas fueron 5812,00 y de acuerdo a un cálculo probabilístico se determinó para el estudio a 85,00 muestras que incluye un adicional de $25 \%$ para contingencia para la caracterización cuantitativa, luego se realizó de manera aleatoria la selección de las viviendas en los 13 sectores del distrito. Para la zonificación se empleó el plano catastral para situar la población e identificar los establecimientos comerciales del distrito que se componen de instituciones educativas, tiendas de abarrotes, establecimientos de venta de fruta y restaurantes, sumando 122 en total tal como se aprecia en la tabla 1, la muestra probabilística en este caso fue 40 establecimientos que incluye un adicional de 10 $\%$. 
Tabla 1

Distribución de vivienda y codificación de las muestras

\begin{tabular}{lccl}
\hline \multicolumn{1}{c}{ Sectores } & Viviendas & $\begin{array}{c}\text { Hogares } \\
(\mathbf{\%})\end{array}$ & Código \\
\hline Yarada Baja & 17 & 17 & AHYBV - 01 al 17 \\
Los Olivos & 11 & 11 & AHLOV - 01 al 11 \\
10 de Mayo & 19 & 19 & AH10MV - 01 al 18 \\
Pueblo Libre & 21 & 21 & AHPLV - 01 al 21 \\
14 de Octubre & 5 & 5 & AH14OCV - 01 al 05 \\
Tito Chocano Olivera & 3 & 3 & AHTCO - 01 al 03 \\
12 de Mayo & 4 & 4 & AH12MV - 01 al 04 \\
Juan Velazco Alvarado & 5 & 5 & JVAV - 01 al 05 \\
Total & 85 & 100 & \\
\hline Nota. Adaptado de información proveniente de Municipalidad La Yarada Los Palos.
\end{tabular}

Tabla 2

Número de establecimientos ubicados en el distrito La Yarada Los Palos

\begin{tabular}{llcc}
\hline Giro & \multicolumn{1}{c}{$\begin{array}{c}\text { Establecimientos } \\
\text { Considerados }\end{array}$} & $\begin{array}{c}\text { Cantidad de } \\
\text { Establecimiento }\end{array}$ & Porcentaje (\%) \\
\hline \multirow{4}{*}{ Comercio } & Tiendas de Abarrotes & 63 & 51,64 \\
& Minimarket & 2 & 1,64 \\
& Ferretería & 1 & 0,82 \\
& Librería & 1 & 0,82 \\
& Mercado de frutas & 12 & 9,84 \\
& Taller mecánica & 1 & 0,82 \\
\multirow{4}{*}{ Servititucional } & Institución Educativa & 6 & 4,92 \\
& Venta de comida & 18 & 14,75 \\
& Grifos & 1 & 0,82 \\
& Cabinas de Internet & 1 & 0,82 \\
& Salón de belleza & 1 & 0,82 \\
& Avenidas & 18 & 14,75 \\
Especiales & Plazas & 1 & 0,82 \\
\hline Total & Ventas de fertilizante & 15 & 12,30 \\
\hline Nota. Adaptado de información proveniente de Municipalidad La Yarada Los Palos.
\end{tabular}

Para el cálculo de la generación Per-Cápita de los residuos sólidos domiciliarios municipales, se determinó mediante el pesaje directo de las muestras que previamente eran codificadas por viviendas de procedencia y clasificado por sectores, para calcular los promedios de generación per cápita por vivienda, se registró los pesos por ocho días, eliminando muestras del primer día para proceder al cálculo, aplica la fórmula 1 y 2 : 
Generación per cápita para cada vivienda.

$$
\begin{aligned}
& \text { GPC Viv } 1=\frac{(P 1 d 2+P 1 d 3+\cdots \ldots+P 1 d 8)}{7 X} \\
& \text { GPC Viv } n=\frac{P 1 d 2+P 1 d 3+\cdots \ldots+P 1 d 8)}{7 Z}
\end{aligned}
$$

\section{Donde:}

GPC Viv1: Generación per-cápita de la vivienda 1

P1d2 : Peso de las bolsas recolectadas de la vivienda día 2

P1d3 : Peso de las bolsas recolectadas de la vivienda día 3

P1d8 : Peso de las bolsas recolectadas de la vivienda día 8

$\mathrm{X} \quad$ : Número de habitantes de la vivienda 1

GPC Vivn: Generación per-cápita de la vivienda $n$

P1d2 : Peso de las bolsas recolectadas de la vivienda día 2

P1d3 : Peso de las bolsas recolectadas de la vivienda día 3

P1d8 : Peso de las bolsas recolectadas de la vivienda día 8

$\mathrm{Z} \quad$ : Número de habitantes de la vivienda $\mathrm{n}$

Para la obtención de la composición Física de los residuos sólidos domiciliarios municipales, se colocaron muestras codificadas en una mesa de madera con forro de plástico color celeste, luego se procedió a homogenizar para luego realizar la caracterización por composición física, se procedió a pesarlos según aspecto y aplicar la fórmula 3, repitiendo el procedimiento por siete días de colecta y por muestra.

$$
\text { Porcentaje } \%=(\mathrm{Pi}) * 100 / \mathrm{Wt}
$$

Donde:

$\mathrm{Pi} \quad$ : Peso de cada Aspecto en los residuos.

Wt : Peso total de los residuos recolectados por día.

La determinación de la densidad de residuos sólidos domiciliarios se realizó empleando un cilindro de $0,14 \mathrm{~m}^{3}$ de capacidad cuyas dimensiones eran altura $87 \mathrm{~cm}$ y el diámetro es $56 \mathrm{~cm}$, el procedimiento consistió en acondicionar en el cilindro con las muestras codificadas y pesadas, eligiendo cada una al azar, seguidamente se elevó el cilindro contiendo los residuos a una altura de $20 \mathrm{~cm}$ del piso, dejándolo caer, esta acción se repitió por tres oportunidades, con la finalidad de uniformizar la muestra en los espacios vacíos del cilindro, para el cálculo se aplicó la fórmula 4 para la determinación densidad.

$$
\text { Densidad }=\frac{W}{V}=\frac{W}{\pi \frac{D^{2} x(H-h)}{4}}
$$

\section{Donde:}

W : Peso de los residuos sólidos

V : Volumen del cilindro

D : Diámetro del cilindro

H : Altura del cilindro 
H : : Altura libre de residuos solidos

$\pi \quad$ : Constante $(3,1416)$

La determinación de la generación Per-Cápita de residuos sólidos no domiciliarios consistió en analizar los residuos generados, en instituciones educativas, restaurantes, abarrotes, tienda de agroquímicos, mercado de frutas, barrido de calle y plaza Los Palos, para ello se aplicó las fórmulas que se muestran en la tabla 3.

\section{Tabla 3}

Resumen de fórmulas para el cálculo de la Generación Per-Cápita de residuos sólidos no domiciliarios

\begin{tabular}{|c|c|c|}
\hline Aspecto calculado & Formula & \\
\hline \multirow{3}{*}{$\begin{array}{l}\text { Generación de residuos sólidos per- } \\
\text { establecimiento } \\
\text { (Kg/establecimiento/día) }\end{array}$} & \multirow{2}{*}{ GPE $=\frac{\mathrm{Kg} . \text { Peso recolectado }}{$\cline { 2 - 3 } \text {$de establecimientos comerciales muestreados }}$} & \multirow[t]{3}{*}{ [5] } \\
\hline & & \\
\hline & & \\
\hline \multirow{2}{*}{$\begin{array}{l}\text { Generación total de residuos por } \\
\text { establecimiento }\end{array}$} & & \multirow{2}{*}{ [6] } \\
\hline & 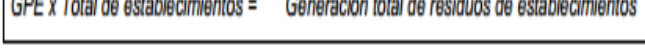 & \\
\hline \multirow{3}{*}{$\begin{array}{l}\text { Generación de los residuos sólidos } \\
\text { municipales por centros de abasto } \\
\text { (kg/mercado/día) }\end{array}$} & \multirow{2}{*}{ GPM $=\frac{K g . \text { Peso recolectado }}{\text { Número de puestos muestreados }}$} & \multirow[t]{3}{*}{ [7] } \\
\hline & & \\
\hline & & \\
\hline $\begin{array}{l}\text { Generación total de los residuos } \\
\text { sólidos en centros de abasto }\end{array}$ & Generación total de residuos en mercados & [8] \\
\hline \multirow{2}{*}{$\begin{array}{l}\text { Generación de residuos sólidos por } \\
\text { Institución Educativa ( } \mathrm{kg} / \text { alumno/día) }\end{array}$} & \multirow{2}{*}{ GPIE $=\frac{K g . \text { Peso recolectado }}{\text { Número de alumnos de Institución Educativa }}$} & \multirow[t]{2}{*}{ [9] } \\
\hline & & \\
\hline \multirow{2}{*}{$\begin{array}{l}\text { Producción de los residuos sólidos } \\
\text { por Institución Educativa }\end{array}$} & & \multirow[t]{2}{*}{ [10] } \\
\hline & GPIE $x$ Total de alumnos en IE = & \\
\hline \multirow{3}{*}{$\begin{array}{l}\text { producción de los residuos sólidos } \\
\text { por restaurantes } \\
\text { (Kg/restaurantes/día) }\end{array}$} & \multirow{3}{*}{$G P R=\frac{K g . \text { Peso recolectado }}{\text { Número de restaurantes muestreados }}$} & \multirow[t]{3}{*}{ [11] } \\
\hline & & \\
\hline & & \\
\hline $\begin{array}{l}\text { Generación total de los residuos } \\
\text { sólidos por restaurantes }\end{array}$ & Generación total de residuos en restaurantes & [12] \\
\hline
\end{tabular}

Nota. Adaptado de la guía MINAM.

\section{Generación total de residuos sólidos no domiciliarios}

Para la determinación de la generación total de los residuos no domiciliarios (GND) se procedió a sumar todos los datos de generación calculadas por actividad económica según la fórmula 13.

$$
\text { GND }=\text { PEtotal }+ \text { GPMtotal }+ \text { GPIEtotal }+ \text { GPRtotal }
$$

Para la determinación de la Composición Física de los Residuos Sólidos no Domiciliarios se aplicó el mismo procedimiento que se aplica para calcular composición física domiciliaria, de forma 
similar en la determinación de la densidad de los residuos sólidos no domiciliarios se emplean las fórmulas para el cálculo de densidad de residuos domiciliaria.

\section{Diseño del relleno sanitario manual}

Se aplicó el procedimiento de la Guía de diseño, construcción, operación, mantenimiento y cierre de relleno sanitario manual del MINAM empleando los resultados obtenidos de la caracterización de residuos y a partir de ello calcular las dimensiones y el número de celdas del relleno sanitario manual.

\section{Resultados}

\section{Generación per cápita (GPC) de los residuos sólidos domiciliarios}

Con la información obtenida sobre caracterización, se calculó la generación per- cápita de residuos sólidos por sectores siendo $0,470 \mathrm{Kg} / \mathrm{hab} /$ día Teniendo en consideración el promedio ponderado de los resultados validos de generación per - cápita de los 7 días de estudio, tal como se muestra en la tabla 4.

\section{Tabla 4}

Generación per cápita (GPC) de los residuos sólidos domiciliarios por sectores

\begin{tabular}{lc}
\multicolumn{1}{c}{ Sectores } & GPC/Sectores (Kg/hab/día) \\
\hline Yarada Baja & 0,380 \\
Pueblo Libre & 0,678 \\
10 de Mayo & 0,483 \\
Los Olivos & 0,350 \\
Tito Chocano Olivera & 0,525 \\
12 de Mayo & 0,431 \\
Juan Velazco Alvarado & 0,300 \\
14 de Octubre & 0,620 \\
\hline GPC Ponderado & 0,470 \\
\hline Nota. Elaboración propia. &
\end{tabular}

Respecto a la composición, en la segregación física de residuos sólidos y el tipo de composición se muestran en el figura 1 donde se puede observar que el 56,77 \% fue de materia orgánica (cascaras de frutas, comida, excrementos de animales, etc.), seguida por residuos sanitarios (papel higiénicos, pañales descartables, toallas higiénicas) con el 11,46\%, inertes (piedra, tierra, polvo) representa el $6,81 \%$, bolsas con el 6,10 \%, botellas de plástico PET el 5,37 \%, metales (latas) el 2,80 \%, papel (periódicos, cartón, colores) el 1,90\%, madera (follajes, hojarascas) representa el $1,70 \%$, el cartón representa $1,43 \%$, otros (material de porcelano) representa 1,04\%, vidrio y tela representa el 0,95\% y 0,92\%, tetrapak y cauchos duro representa el 0,51\%, pilas representa el 0,23 $\%$, finalmente restos de medicina y tecnopor representa el $0,20 \%$ del total, los porcentaje representa el $100 \%$ del distrito La Yarada Los Palos. 


\section{Figura 1}

Composición Física de los Residuos Sólidos domiciliarios

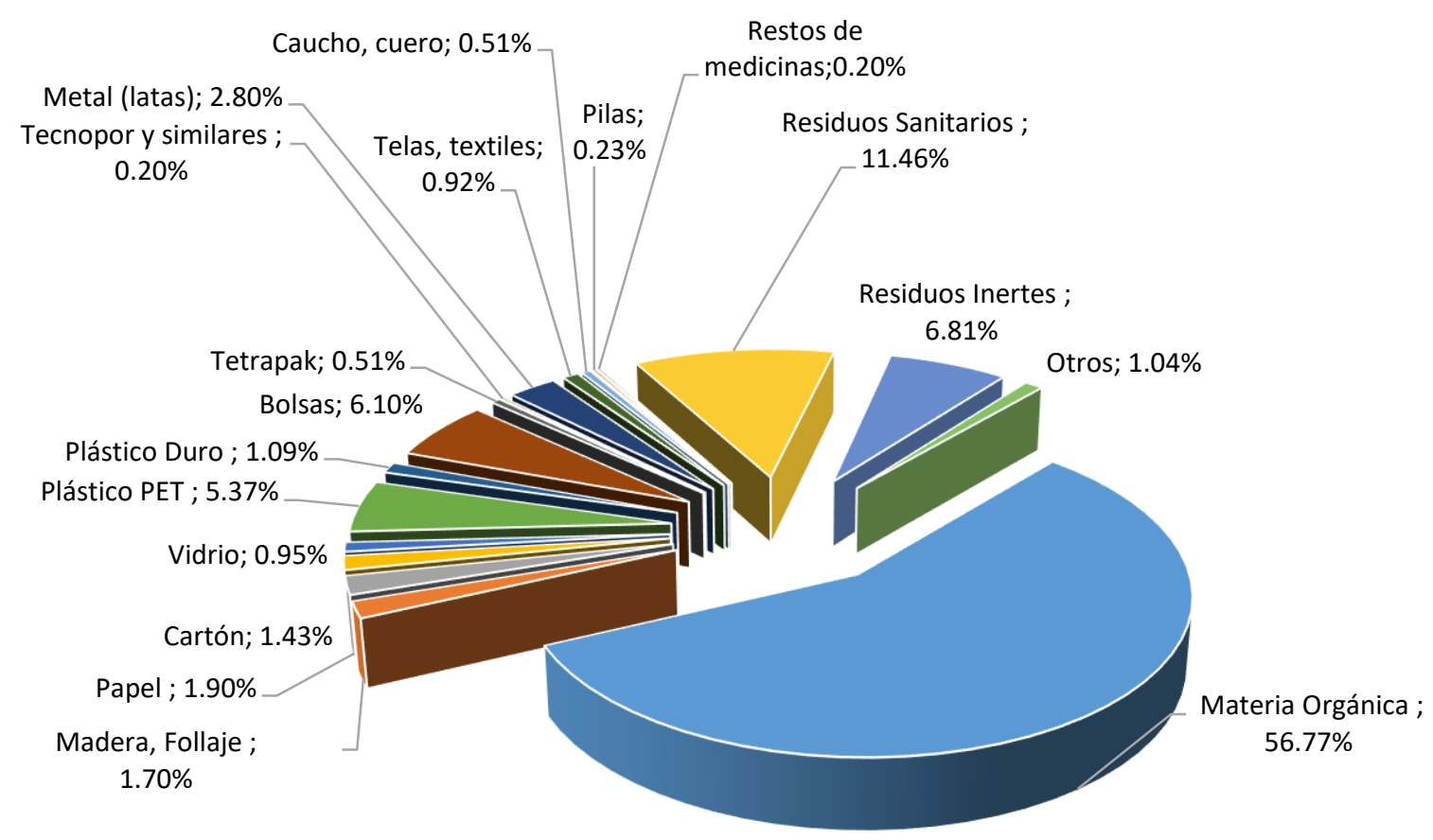

Nota. Elaboración propia.

Se determinó la densidad promediando de los datos obtenidas por los siete días consecutivos, sin compactar para cada uno de los sectores del distrito, cuyo valor es $221,341 \mathrm{~kg} / \mathrm{m}$

\section{Características de residuos no domiciliarios}

En la figura 2 se puede observar que los colegios son los que generan más residuos sólidos con $38,47 \%$ además las tiendas de abarrotes generan $28,53 \%$ mientras que las tiendas de frutas y calles generan el $8,60 \%$, así mismo el 7,21 \% es generado por restaurantes y finalmente las plazas con el $4,10 \%$. 


\section{Figura 2}

Tipos de establecimiento según la generación de residuos solidos

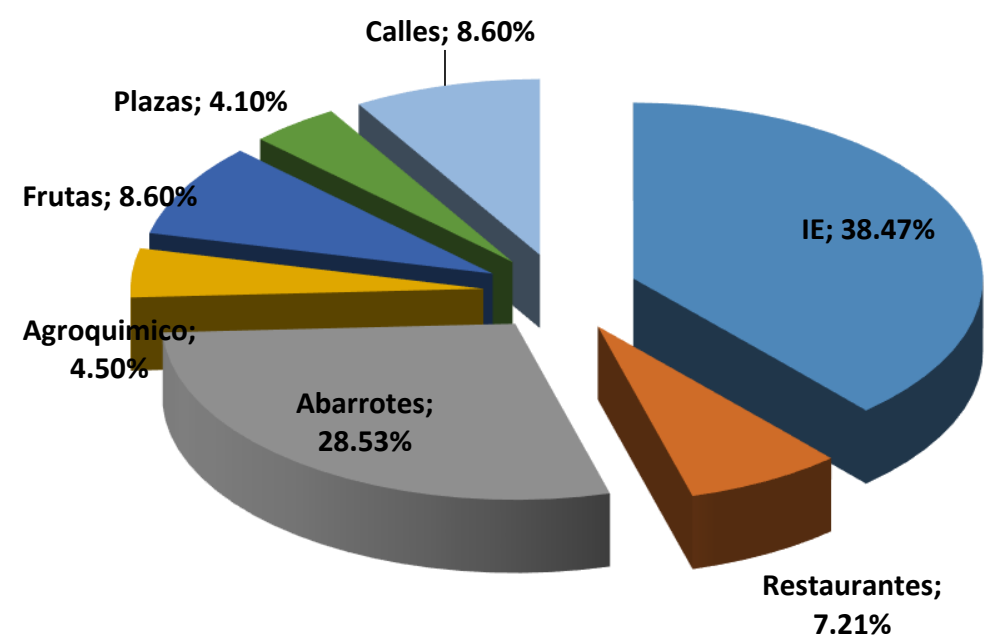

Nota. Elaboración propia.

En la figura 3 se observa que el 42,94 \% de residuos sólidos generados está conformado por materia orgánica, seguido de 10,59 \% que se compone de bolsas, el 8,88 \% de botellas plásticas PET, el $8,80 \%$ de establecimientos genera cartón, un 8,07 \% genera residuos inertes (casi el $100 \%$ de la población no cuenta con asfaltado en sus viviendas) es por ello la acumulación de residuos inerte, el $4,58 \%$ de los establecimientos generan residuos sanitarios (papel higiénicos, pañales, etc.), un 4,63 $\%$ genera papel (como recibos), aproximadamente el $3 \%$ generan residuos de plástico duro y vidrio, finalmente menos del $1 \%$ se genera residuos de (medicina, pilas, latas, tecnopor, telas, caucho y cuero), el 1,42\% de los establecimientos genera follaje o hojarasca.

La densidad de los residuos sólidos generados en los establecimientos fue; densidad de residuos sueltos $91,989 \mathrm{~kg} / \mathrm{m}^{3}$, la densidad compactada promedio $129,531 \mathrm{~kg} / \mathrm{m}^{3}$, y el grado de compactación 1,170, como la proporción de la reducción posible de volumen por compactación y su almacenamiento o disposición final.

\section{Diseño de Relleno Sanitario Manual}

Para la elaboración del diseño del relleno sanitario, se empleó los criterios establecidos en la guía del MINAM denominada "Guía de Diseño, Construcción, Operación, Mantenimiento y Cierre de Relleno Sanitario Manual" procediéndose a estimar un tiempo de vida útil de 10 años, según se puede apreciar en la tabla 7. La proyección se estimó para el periodo de 10 años, considerando un incremento poblacional del $1,7 \%$ de acuerdo al censo del INEI (2017) 
Figura 3

Composición Física de los RRSS no domiciliarios

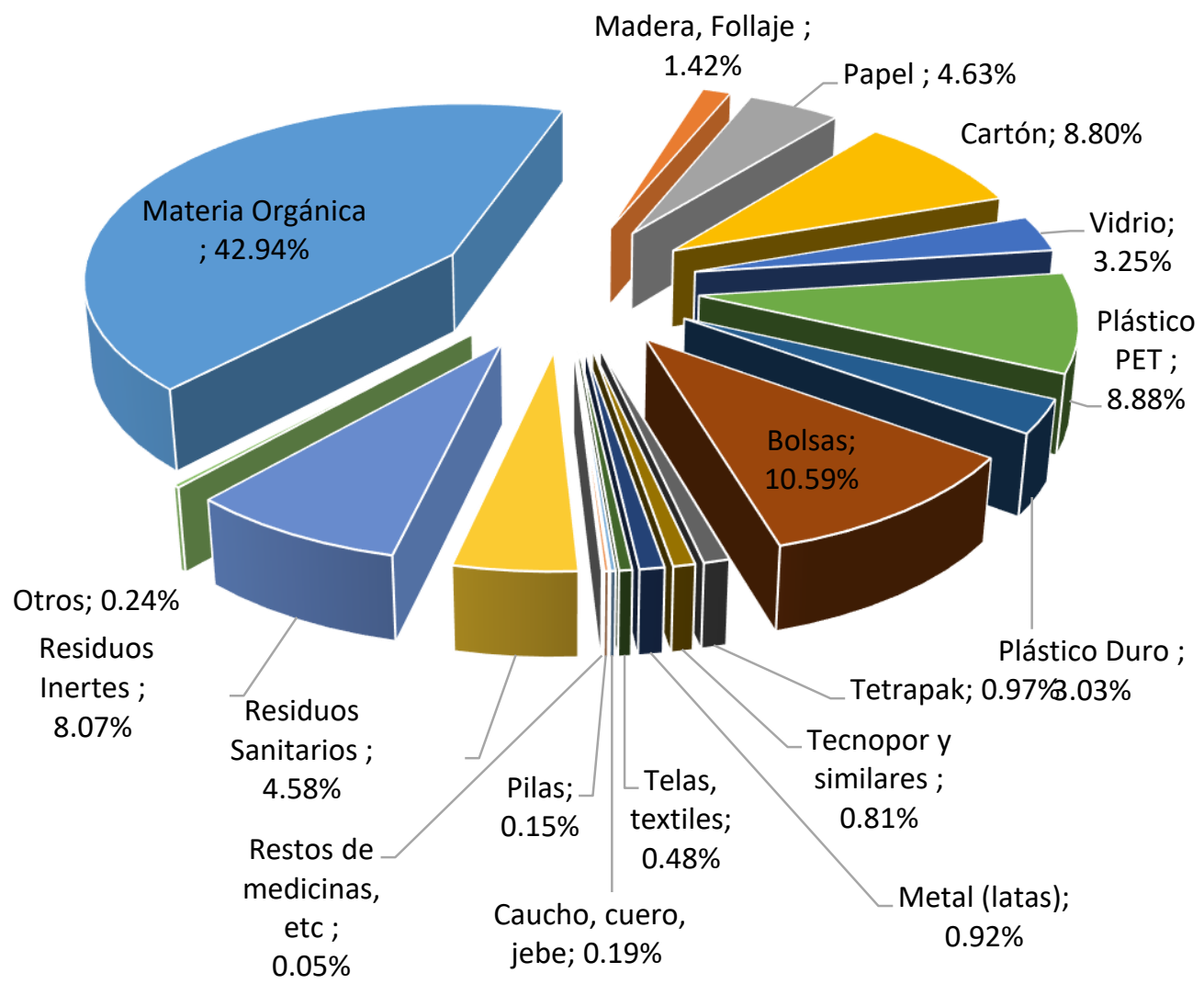

Nota. Elaboración propia.

Tabla 7

Proyección de la generación de residuos

\begin{tabular}{|c|c|c|c|c|c|c|c|c|c|c|c|c|c|}
\hline ؟̊ำ & $\begin{array}{l}\text { :0 } \\
\frac{\pi}{0} \\
\frac{\pi}{0}\end{array}$ & 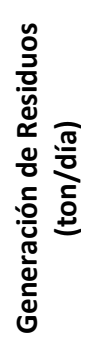 & 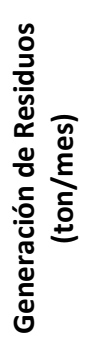 & 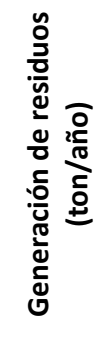 & 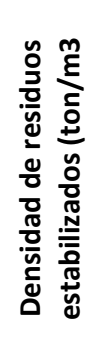 & 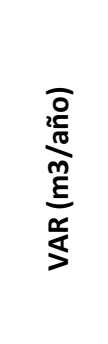 & 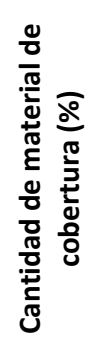 & 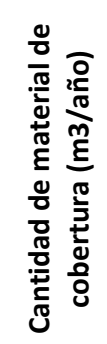 & 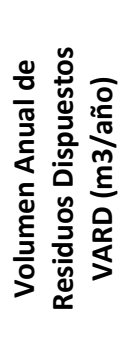 & 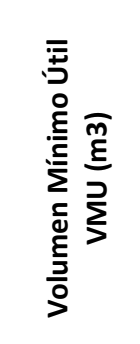 & 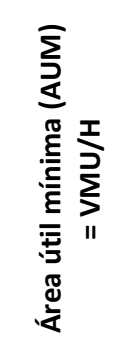 & 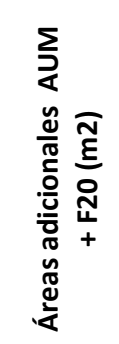 & 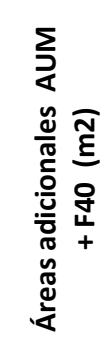 \\
\hline 0 & 5812 & 2,73 & 81,9 & 983,4 & 0,600 & 1639,0 & & & & & & & \\
\hline 1 & 5911 & 2,78 & 83,3 & 1000,1 & 0,600 & 1666,8 & 25 & 416,71 & 2083,56 & 22503,96 & 9376,65 & 11252,0 & 13127,3 \\
\hline 2 & 6011 & 2,83 & 84,8 & 1017,1 & 0,600 & 1695,2 & 25 & 423,80 & 2118,98 & & & & \\
\hline 3 & 6113 & 2,87 & 86,2 & 1034,4 & 0,600 & 1724,0 & 25 & 431,00 & 2155,00 & & & & \\
\hline 4 & 6217 & 2,92 & 87,7 & 1052,0 & 0,600 & 1753,3 & 25 & 438,33 & 2191,64 & & & & \\
\hline 5 & 6323 & 2,97 & 89,2 & 1069,9 & 0,600 & 1783,1 & 25 & 445,78 & 2228,89 & & & & \\
\hline 6 & 6431 & 3,02 & 90,7 & 1088,1 & 0,600 & 1813,4 & 25 & 453,36 & 2266,79 & & & & \\
\hline 7 & 6540 & 3,07 & 92,2 & 1106,6 & 0,600 & 1844,3 & 25 & 461,06 & 2305,32 & & & & \\
\hline 8 & 6651 & 3,13 & 93,8 & 1125,4 & 0,600 & 1875,6 & 25 & 468,90 & 2344,51 & & & & \\
\hline 9 & 6764 & 3,18 & 95,4 & 1144,5 & 0,600 & 1907,5 & 25 & 476,87 & 2384,37 & & & & \\
\hline 10 & 6879 & 3,23 & 97,0 & 1164,0 & 0,600 & 1939,9 & 25 & 484,98 & 2424,90 & & & & \\
\hline
\end{tabular}

Nota. Elaboración propia. 
Seguidamente se calculó el volumen anual de los residuos dispuestos al relleno sanitario manual, tal como se puede observar en la tabla 8, con una proyección de 10 años contando a partir del año 0 , en generación de residuos en Ton/año, residuos compactados en el relleno sanitario, los residuos acumulados, el material de cobertura, VARD acumulados en $\mathrm{m}^{3}$.

Se consideró una baja del volumen de los residuos domiciliarios dispuestos por su compactación hasta lograr una densidad promedio de $0,6 \mathrm{Ton} / \mathrm{m}^{3}$. Respecto al material de cobertura tendrá un volumen de $25 \%$ en referencia del volumen de residuos compactados

La proyección a 10 años da como resultado la generación de 24552.7 m3 de residuos sólidos como volumen anual de residuos sólidos acumulado.

Finalmente se calculó la capacidad útil de diseño, los parámetros son; Largo superior de 60 metros, el ancho superior 30 metros, el área superior $1800 \mathrm{~m} 2$, la altura 2,4 m, Talud de la trinchera (H) la cantidad es de 1, el talud de la trinchera (V), largo inferir $55.2 \mathrm{~m}$, Ancho inferior 25,2 m, área inferior es de 512,04 m2, con un volumen útil (VUD) de 3829,2 $\mathrm{m}^{3}$.

Según el cálculo se requiere la apertura de 6 zanjas de 3829,2 $\mathrm{m}^{3}$ para cumplir con los de 24 552,7 $\mathrm{m}^{3}$ de VARD proyectados al 2027.

\section{Tabla 8}

Volumen anual de residuos dispuestos (VARD)

\begin{tabular}{cccccc}
\hline Año & $\begin{array}{c}\text { Generación } \\
\text { de residuos } \\
\text { (ton/año) }\end{array}$ & $\begin{array}{c}\text { Residuos } \\
\text { compactados } \\
\text { en el relleno } \\
\text { sanitario }(\mathbf{m} 3)_{\mathbf{1}}\end{array}$ & $\begin{array}{c}\text { Residuos } \\
\text { compactados } \\
\text { acumulados } \\
(\mathbf{m} 3)_{\mathbf{1}}\end{array}$ & $\begin{array}{c}\text { Material de } \\
\text { cobertura } \\
\mathbf{( m 3})_{\mathbf{2}}\end{array}$ & $\begin{array}{c}\text { VARD } \\
\text { acumulado } \\
\text { (m3) }\end{array}$ \\
\hline 2017 & 983,4 & 1639,0 & 1639,0 & 409,7 & 2048,7 \\
2018 & 1000,1 & 1666,8 & 3305,8 & 826,5 & 4132,3 \\
2019 & 1017,1 & 1695,2 & 5001,0 & 1250,3 & 6251,3 \\
2020 & 1034,4 & 1724,0 & 6725,0 & 1681,3 & 8406,3 \\
2021 & 1052,0 & 1753,3 & 8478,3 & 2119,6 & 10597,9 \\
2022 & 1069,9 & 1783,1 & 10261,4 & 2565,4 & 12826,8 \\
2023 & 1088,1 & 1813,4 & 12074,9 & 3018,7 & 15093,6 \\
2024 & 1106,6 & 1844,3 & 13919,1 & 3479,8 & 17398,9 \\
2025 & 1125,4 & 1875,6 & 15794,7 & 3948,7 & 19743,4 \\
2026 & 1144,5 & 1907,5 & 17702,2 & 4425,6 & 22127,8 \\
2027 & 1164,0 & 1939,9 & 19642,2 & 4910,5 & 24552,7 \\
\hline
\end{tabular}

Nota. Elaboración propia. 


\section{Tabla 9}

Cálculo de la capacidad útil de diseño

\begin{tabular}{|c|c|c|}
\hline Parámetro/Fórmula & $\begin{array}{l}\text { Unidad de } \\
\text { medida }\end{array}$ & Cantidad \\
\hline Largo superior (Is) & $\mathrm{m}$ & 60 \\
\hline Ancho superior (as) & $\mathrm{m}$ & 30 \\
\hline Área superior $(A s)=$ ls $x$ as & $\mathrm{m} 2$ & 1800 \\
\hline Altura $=\mathrm{h}$ & $\mathrm{m}$ & 2,4 \\
\hline Talud de la trinchera $(\mathrm{H})$ & & 1 \\
\hline Talud de la trinchera (V) & & 1 \\
\hline largo inferior $(\mathrm{li})=\mathrm{Is}-2 \times \mathrm{hH}$ & $\mathrm{m}$ & 55,2 \\
\hline Ancho inferior (ai) $=$ as $-2 \times \mathrm{hV}$ & $\mathrm{m}$ & 25,2 \\
\hline Area Inferior $(A i)=l i x$ ai & $\mathrm{m} 2$ & 1391,04 \\
\hline $\operatorname{VUD}=(A s+A i) / 2 * h$ & m3 & 3829,2 \\
\hline
\end{tabular}

Nota. Elaboración propia.

\section{Discusiones}

La generación per cápita de residuos sólidos domiciliarios en el distrito La Yarada Los Palos es de 0,470 kg/hab-día, ello implica que la generación domiciliaria distrital anual para el 2018 tuvo un incremento de 16926,480 kg/año de residuos sólidos. La producción per cápita promedio encontrada en el distrito de La Yarada Los Palos está por inferior de la determinada en el Distrito de Tacna (0,49 Kg/hab/día) y la del nivel nacional (0,53 Kg/hab./día) según el MINAM (2008).

En la composición física de residuos sólidos del distrito La Yarada Los Palos se determinó que el mayor porcentaje es la materia orgánica $(56,77 \%)$ que podría ser una alternativa para uso tales como el compostaje y la obtención de otros sub productos.

Respecto a la densidad $(224,682 \mathrm{Kg} / \mathrm{m} 3)$, se encuentra ligeramente inferiores al rango nacional, nos demuestra la importante generación de residuos orgánicos, cifras concordantes con las halladas a nivel nacional cuyo rango es de 230 a $350 \mathrm{Kg} / \mathrm{m} 3$ MINAM (2008)

Para establecimiento comerciales o diferentes tipos de giros no domiciliarios en el distrito La Yarada Los Palos, se determinó que en las instituciones educativas es de $38,47 \%$ del total de residuos sólidos generados del distrito. Estos resultados se reflejarían por no existir educación ambiental o un plan de manejo de residuos sólidos dentro de la institución que haga que los residuos generados por personal administrativo y alumnos disminuyan o en todo caso sea reciclado.

La composición física no domiciliarios está conformada por materia orgánica con el 42,94 \% de acumulación de compostables, mientras la densidad de los residuos sueltos es de $91,989 \mathrm{~kg} / \mathrm{m}^{3}$, y la densidad compactada es de $129,531 \mathrm{~kg} / \mathrm{m}^{3}$, lo cual indicaría que reducción de volumen es posible por la utilización de equipamiento rural con compactación para el almacenamiento público, con un grado de compactación aproximado de 1 a 1,170.

De acuerdo a los datos registrados en la generación diaria de residuos 2,73 Tn/día corresponde el diseño de un relleno sanitario manual (>50 Tn/día), lo cual concuerda con las características rurales del distrito, así mismo las medidas de segregación en fuente y aprovechamiento se podría reducir el VARD al 2027 de 24 552,7 m³ según lo indicado por MINAM. 


\section{Conclusiones}

La generación per cápita de residuos sólidos domiciliarios en el distrito La Yarada Los Palos es de $0,470 \mathrm{~kg} / \mathrm{hab}$-día.

La composición física de residuos sólidos con mayor porcentaje es la materia orgánica (56,77\%), mientras que la densidad promedio de viviendas en el estudio de caracterización sin compactar es de $224,682 \mathrm{~kg} / \mathrm{m}^{3}$ y compactado es de $305,664 \mathrm{~kg} / \mathrm{m}^{3}$.

Para establecimiento comerciales o diferentes tipos de giros no domiciliarios, la generación per - cápita de las instituciones educativas es de 0,088 kg/alumno/día, en los establecimientos de restaurantes de 2,706 kg/ restaurante/día, en los establecimientos de abarrotes la generación per cápita de 0,838 kg/tienda/día, la generación de residuos en mercado por puesto es 2,198 $\mathrm{kg} /$ puesto/día, la GPCBC es de $2,353 \mathrm{~kg} / \mathrm{km} / \mathrm{día}$, la generación per cápita por tiendas de agroquímicos es de 0,687 kg/tienda/día.

La composición física no domiciliarios está conformada por materia orgánica con el 42,94 \%, mientras que la densidad suelta promedio es $91,89 \mathrm{~kg} / \mathrm{m}^{3}$ sin compactar y compactado de 129,531 $\mathrm{kg} / \mathrm{m}^{3}$.

El diseño del relleno sanitario estará conformado por seis zanjas de $3829,2 \mathrm{~m}^{3}$ para un VARD de $24552,7 \mathrm{~m}^{3}$ de residuos sólidos. El relleno sanitario deberá contar con un área comprendido entre $11252,0-13127,3 \mathrm{~m}^{2}$.

\section{Referencias Bibliográficas}

MINAM. (2018). Guía para la caracterización de residuos sólidos municipales

MINAM. (2008). Informe de la Situación Actual de la Gestión de Residuos Sólidos Municipales. [En línea]: (http:// www.minam.gob.pe, 30 Ag. 2011).

MINAM. Guía de diseño, construcción, operación, mantenimiento y cierre de relleno sanitario manual. Red de Instituciones especializadas en capacitación para la gestión integral de los residuos sólidos.

Puerta Echeverri, S. M. (2004). Los residuos sólidos municipales como acondicionadores de suelos

Rendón, A. F. M. (2012). Caracterización de residuos sólidos. Cuaderno activa, 4, 67-72.

.Ruiz Morales, M. (2012). Caracterización de residuos sólidos en la Universidad Iberoamericana, Ciudad de México. Revista internacional de contaminación ambiental, 28(1), 93-97.

Sáez, A., \& Urdaneta, J. A. (2014). Manejo de residuos sólidos en América Latina y el Caribe. Omnia, 20(3), 121-135. 